\title{
Creación de contextos de aprendizaje crítico para la mejora del aprendizaje del Proceso de Enfermería
}

\section{Creating critical learning contexts to improve the Nursing Process learning}

Regina AlLande Cussó

Departamento de Enfermería

Universidad de Sevilla

rallande@us.es

ORCID: https://orcid.org/0000-0001-8325-0838

Fecha de recepción:

Fecha de aceptación:

DOI: http://dx.doi.org/10.12795/9788447221912.053

Pp.: $1224-1240$ 


\section{RESUMEN}

En este capítulo se describe el Ciclo de Mejora en el Aula (CIMA) llevado a cabo durante el curso académico 2019-20 en los seminarios de la asignatura de Historia, Teoría y Métodos en Enfermería II, del Grado en Enfermería de la Universidad de Sevilla, en el contexto del programa de Formación e Innovación Docente del Profesorado (FIDOP) de la misma Universidad. El diseño del CM respondió a un proceso de enseñanza-aprendizaje basado en la implementación de la simulación de casos y el análisis de imágenes, en el que participaron 26 estudiantes. Se llevó a cabo una evaluación de contenidos mediante un diseño test-retest. Los resultados obtenidos fueron positivos, por lo que cabe concluir que el CM consiguió los objetivos propuestos respecto a la adquisición de los contenidos tratados.

PALABRAS CLAVE: Historia, teoría y métodos en Enfermería II; Grado en Enfermería; Docencia universitaria; experimentación docente universitaria; simulación.

\section{ABSTRACT}

This chapter describes an Improvement Cycle in Classroom, carried out during 2019-20, in the context of History, Theory and Methods in Nursing II subject, of the Degree in Nursing at the University of Seville. Moreover, the Cycle is included in a Teaching Innovation Program for Professors at the same University. The design of the Cycle is related to a teaching-learning process based on the implementation of case simulation and image analysis, in which 26 students participated. A content evaluation was carried out using a test-retest design. The results obtained were positive, so it can be concluded that the Improvement Cycle achieved the objectives regarding the acquisition of the contents treated.

KEYWORDS: History, theory and Methods in Nursing II; Nursing Degree; University education; University education test; simulation. 


\section{Contexto educativo}

Los seminarios de la asignatura "Historia, Teoría y Métodos de Enfermería II" son de carácter obligatorio, y tienen asignados una carga de 2.2 créditos en el plan docente del Grado en Enfermería (Departamento de Enfemería de la Universidad de Sevilla, 2019; Departamento de Enfermería de la Universidad de Sevilla, 2019). Al respecto, la competencia 3.13 del plan docente de la asignatura de Historia, teoría y Métodos de la Enfermería II (HTM II) especifica que el estudiante debe aplicar el proceso de enfermería para proporcionar y garantizar el bienestar, calidad y seguridad de la persona atendida (Departamento de Enfermería de la Universidad de Sevilla, 2019). Además, de entre las competencias genéricas, de describe la capacidad de aplicar la teoría a la práctica, la resolución de problemas, y el trabajo en equipo (Departamento de Enfermería de la Universidad de Sevilla, 2019). Así mismo, el estudiante no ha participado anteriormente, ni participa, en prácticas clínicas, durante el transcurso de la asignatura de HTM II, puesto que la misma queda adscrita en la organización docente del 20 curso 1er cuatrimestre, y las prácticas clínicas se inician en el 2 o cuatrimestre (Departamento de Enfemería de la Universidad de Sevilla, 2019).

Dado este contexto, el discente tiene dificultades para comprender y analizar a los protagonistas de los casos problema que se le plantean, en todas sus esferas, y desde una perspectiva enfermera; esto es, el estudiante no puede "hacerse una idea" de la persona a la que debe cuidar mediante la implementación de un Proceso de Enfermería. Ello, dificulta el proceso de enseñanza-aprendizaje del mismo, que además es el objetivo general de la asignatura. Por ello, cabe diseñar una propuesta de mejora docente que incluya la creación de contextos de aprendizaje lo más realistas posibles, que permitan al estudiante integrar y poner en valor el Proceso de Enfermería (Bain, 2007). 


\section{Mapa de contenidos}

La figura 1 representa el mapa de contenidos organizadores, procedimentales, intelectuales y actitudinales que se pretende que el estudiante adquiera tras la finalización del CIMA (Agencia Nacional de Evaluación de la Calidad y Acreditación, 2004, 2008; Alfaro, 2003; Cibanal y Arce, 2009; Fernandez, Navarro, y Luis, 2005; Wilkinson, 2012):

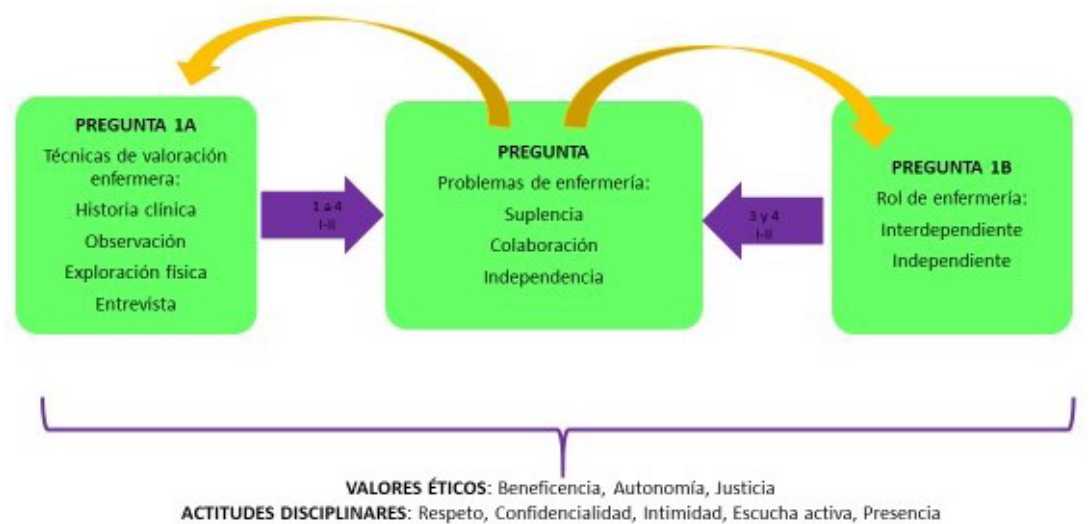

Figura 1 Mapa de contenidos y problemas

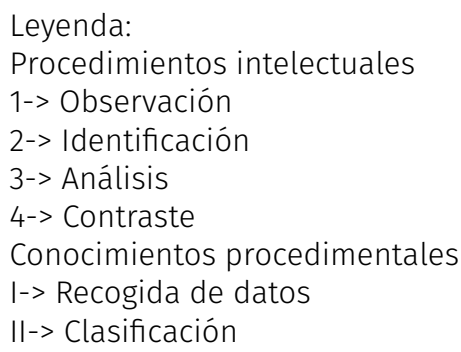

La pregunta clave que orientó el diseño y desarrollo del modelo metodológico fue: ¿Qué tipología de problemas de enfermería existen?. A partir de ella, se plantearon dos preguntas más:
a) Pregunta 1A: ¿Qué técnicas de valoración existen para identificar los problemas de enfermería?
b) Pregunta 1B: ¿Cuál es el rol y la responsabilidad del profesional de enfermería en ellos?




\section{Modelo metodológico}

El CIMA diseñado tiene una duración total de 8 horas, y se llevó a cabo en dos partes, con una muestra de 26 estudiantes. El modelo metodológico empleado se muestra en la figura 2:

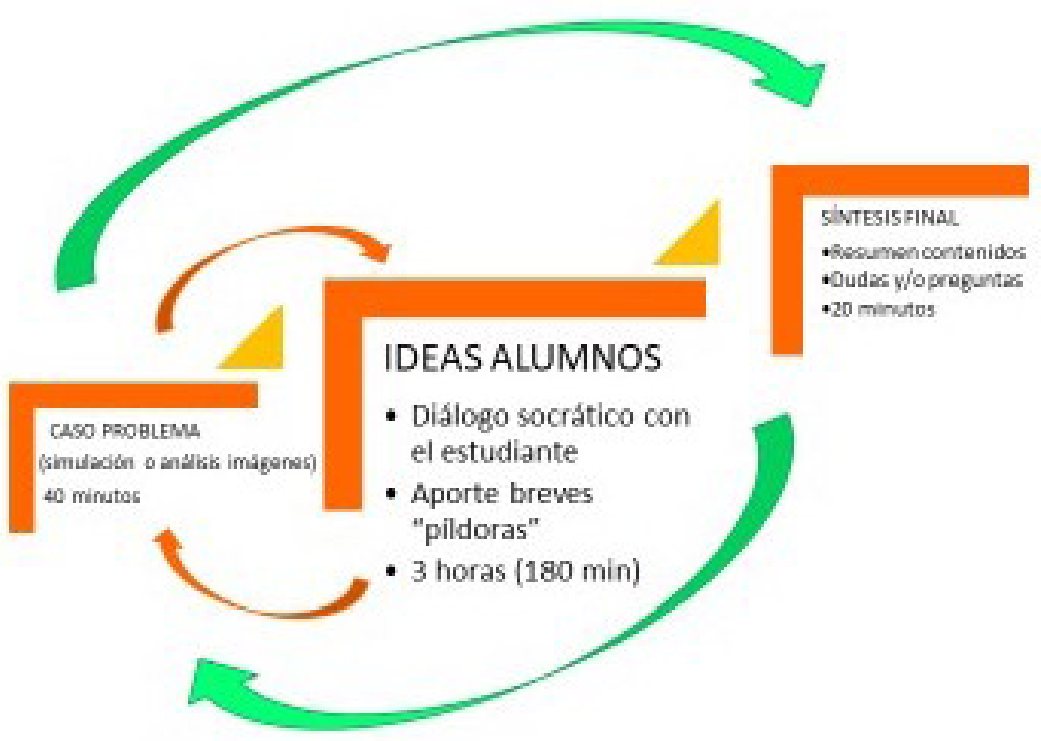

Figura 2 Modelo metodológico del ciclo de mejora

El rol del docente fue de guía en el aprendizaje de los contenidos, a partir de un diálogo socrático con los estudiantes, y de la aportación de pequeñas "píldoras informativas" necesarias para la correcta comprensión de los contenidos.

Las primeras 4 horas del CM se destinaron a dar respuesta a la pregunta clave por parte del estudiante. Durante las siguientes 4 horas, se procedió a dar respuesta a las preguntas 1A y 1B. Así, la ejecución del modelo metodológico propuesto, desde el planteamiento de un caso problema hasta la síntesis, se llevó a cabo implementándolo dos veces al completo. 
En relación al caso problema, se utilizó la técnica de la simulación y también de análisis de imágenes para la presentación del mismo. Tras ello, los estudiantes fueron trabajando las actividades docentes diseñadas. Éstas conectaron siempre las ideas de los alumnos/as con los datos objetivables en el caso-problema. Finalmente, se llevó a cabo un proceso de análisis de los contenidos aprendidos y sintesis de los mismos.

Cabe mencionar que los estudiantes fueron convenientemente informados de su participación en un CM de la docencia en la asignatura, como parte del programa FlDOP de la Universidad de Sevilla, sin que ello afectara al contenido del programa docente ni a su evaluación final.

\section{Diseño de actividades docentes}

La secuencia de actividades programadas, con el objetivo de dar respuesta a las preguntas planteadas, es la siguiente:

\subsection{Actividades docentes de la primera parte del CIMA (4 horas)}

ACTIVIDAD 1 Tiempo: 10 minutos

DESCRIPCIÓN:

- Proporcionar a cada estudiante una copia del caso 1.

- Lectura individual.

OBJETIVOS:

- Activar la actividad cognitiva del estudiante

- Facilitar la comprensión de la situación descrita en el caso-problema por parte del estudiante

RECURSOS: 26 copias del caso-problema

FASE DEL MODELO METODOLÓGICO: Caso-Problema

ACTIVIDAD 2 Tiempo: 20 minutos

Jornadas de Formación e Innovación Docente del Profesorado | № 2 (2019) Esta obra se distribuye con la licencia Creative Commons Reconocimiento-NoComercial-SinObraDerivada 
DESCRIPCIÓN:

- Trabajo grupal: crear sub-grupos de trabajo

- Planteamiento de la pregunta clave no 1

- Identificar la técnica de valoración de enfermería utilizada para recabar los datos descritos en el caso-problema (trabajo en sub-grupos)

OBJETIVO: Implicar al estudiante en la construcción del conocimiento específico, vinculando teoría y práctica.

FASE DEL MODELO METODOLÓGICO: Ideas de los alumnos

\begin{tabular}{|l|}
\hline ACTIVIDAD 3 Tiempo: 10 minutos \\
\hline DESCRIPCIÓN: \\
Puesta en común de las respuestas de los estudiantes a la pregunta clave \\
no 1 \\
Consenso final respecto a las técnicas de valoración empleadas y los da- \\
tos reflejados en el caso-problema. \\
OBJETIVO: Fomentar la capacidad de identificación de categorías abstractas \\
y de síntesis. \\
FASE DEL MODELO METODOLÓGICO: Ideas de los alumnos
\end{tabular}

ACTIVIDAD 4 Tiempo: 180 minutos

DESCRIPCIÓN:

- Actividad de simulación:

a) Cada sub-grupo "construirá el personaje" protagonista del problema-caso, utilizando un muñeco simulador -sobre el que podrá colocar aquellos elementos necesarios para su caracterización, y que estarán disponibles en el aula- (30 minutos)

La docente guiará dicha construcción con el aporte de "píldoras informativas": modelos teóricos que guían la práctica, valores éticos, actitudes disciplinares, relación enfermera-paciente.

b) Consenso común de la mejor construcción del personaje (10 minutos)

c) A partir de la caracterización del protagonista del caso-problema elegida, la docente trabajará los contenidos a partir de diferentes preguntas dirigidas el grupo clase (140 minutos):

- ¿Cómo es el proceso de valoración?

- ¿Qué valores éticos deben fundamentarlo?

- ¿Qué actitudes y conductas deben demostrarse?

- ¿En qué momento empieza la fase de diagnóstico?

- ¿Los datos recogidos deben ser organizados o clasificados?

- ¿En qué categorías los podemos clasificar?

- ¿Es importante realizar un análisis de los datos clasificados?

- Aporte de "píldoras informativas": aspectos legales y disciplinares en torno al constructo "interacción de cuidado", herramientas de clasificación de datos

- Uso de la técnica "visual thinking" para plasmar las ideas de los estudiantes en la pizarra. 
OBJETIVO:

- Fomentar la capacidad de análisis, contraste, identificación, y observación del estudiante

- Poner en valor aspectos ético-legales de la disciplina enfermera RECURSOS: muñeco simulador, material para caracterizarlo (sonda nasogástrica, vía periférica, adhesivo fijador, sistema para suero fisiológico (SF), soporte para SF, sábana), pizarra.

FASE DEL MODELO METODOLÓGICO: Caso-problema e ideas de los alumnos

ACTIVIDAD 5 Tiempo: 20 minutos

DESCRIPCIÓN:

- Sintesis de la primera parte del CM por parte del grupo clase: ideas y conceptos aprendidos

- Resolución de dudas o preguntas

- Síntesis parcial por parte del docente, respecto al total de los contenidos del CM.

OBJETIVO: Fomentar la capacidad de análisis y sintesis del estudiante en relación a los conceptos aprendidos

FASE DEL MODELO METODOLÓGICO: Síntesis

\subsection{Actividades docentes de la segunda parte del CIMA ( 4 horas)}

ACTIVIDAD 1 Tiempo: 40 minutos

DESCRIPCIÓN:

- Explicación y descripción de la tarea y sus objetivos

- Planteamiento de la pregunta clave no 2

- Visualización del vídeo propuesto como caso-problema

- Identificación de los diferentes problemas de enfermería observados, así como el rol del profesional en cada uno de ellos.

- Realizar un esquema respecto a la naturaleza de los problemas de enfermería detallados en el caso, comprendiendo la perspectiva del Modelo Bifocal de Práctica Clínica de Carpenito (Carpenito, 2009), así como la aplicación de la teoría conceptual de Henderson al Proceso de Enfermería, que realiza María Teresa de Luis (Fernandez y cols, 2005).

OBJETIVO: Activar la actividad cognitiva del estudiante y la memoria respecto a los modelos teóricos vistos en clase, a través de la clasificación de problemas enfermeros en categorías diferentes según la teoría que guíe la práctica.

FASE DEL MODELO METODOLÓGICO: Caso Problema e ideas de los alumnos 
ACTIVIDAD 2 Tiempo: 180 minutos

DESCRIPCIÓN:

- Visualización de imágenes (entre 10 y 15) de personas con una situación de salud- enfermedad determinada

- A partir de cada imagen, los estudiantes aportarán sus ideas en respuesta

a las siguientes preguntas formuladas por la docente:

a) ¿Qué problemas son de naturaleza interdependiente?

b) ¿De quién es la responsabilidad del tratamiento o intervención sobre los mismos?

c) ¿Qué problemas son de naturaleza independiente?

d) ¿Qué problemas detectados son de "independencia" según el modelo de Henderson?

e) ¿Qué problemas detectados son de "autonomía" según la adaptación de María Teresa de Luis respecto el modelo de Henderson?

f) ¿Qué problemas detectados son de "colaboración" según la adaptación de María Teresa de Luis respecto el modelo de Henderson?

- Aporte de "píldoras informativas": modelos teóricos, roles de enfermería, naturaleza de los problemas de enfermería, práctica enfermera guiada por la teoría

OBJETIVO: Afianzar los modelos teóricos que guían la práctica clínica, y los roles del profesional de enfermería.

FASE DEL MODELO METODOLÓGICO: Caso-problema e ideas de los alumnos

ACTIVIDAD 3 Tiempo: 20 minutos

DESCRIPCIÓN

- Sintesis de la segunda parte del CIMA por parte del grupo clase: ideas y conceptos aprendidos

- Resolución de dudas o preguntas

- Sintesis final por parte del docente, respecto al total de los contenidos del CIMA.

OBJETIVO: Fomentar la capacidad de análisis y síntesis del estudiante en relación a los conceptos aprendidos

FASE DEL MODELO METODOLÓGICO: Síntesis

\section{Desarrollo de las sesiones ciclo de mejora}

La puesta en marcha de las actividades docentes, planificadas para dar respuesta a la pregunta clave, se desarrolló sin incidencia alguna y según el diseño de las mismas. Los estudiantes participaron en cada una de las 
tareas propuestas de forma proactiva y colaboradora. Así, la posibilidad de construir el "personaje-paciente" mediante un muñeco simulador, a partir de los datos facilitados en el caso-problema, fue acogida con ilusión y agrado por parte del alumnado. Además, el uso de la simulación en una asignatura que demanda "valorar a la persona en su situación de salud actual" y emitir un juicio respecto a sus problemas de enfermería, facilita que el estudiante comprenda esa situación concreta de salud. Por otro lado, el visionado del vídeo e imágenes, como herramientas para dar respuesta a la pregunta $1 \mathrm{~A}$ y $1 \mathrm{~B}$, fueron percibidas como facilitadoras del aprendizaje de los contenidos de la asignatura por parte del grupo de estudiantes.

El rol docente fue siempre de guía en el aprendizaje, mostrando actitud de disponibilidad total, así como atención plena al desarrollo de las tareas que debían abordarse en los sub-grupos de trabajo.

Cabe mencionar la dificultad de los discentes para reagruparse en grupos de trabajo más pequeños de forma autónoma; esto es, los estudiantes presentaron ciertas dificultades para sub-dividirse en grupos de trabajo en el aula. Así, este contenido procedimental, que no estaba incluido en el mapa de contenidos del CIMA, será incorporado como contenido de próximos ciclos de mejora de la asignatura.

Desde un punto de vista analítico, y según las impresiones de los discentes, sería pertinente considerar el aumento de horas de simulación, incluso incorporar actividades de observación de personas reales en un contexto sanitario. Con ello, podríamos crear espacios de aprendizaje crítico naturales, que ayuden al estudiante a comprender la situación real de salud de la persona, desde la perspectiva disciplinar de enfermería -en un nivel del Grado en Enfermería, como es 2 - curso 1er cuatrimestre, en el que los alumnos todavía no han iniciado sus prácticas clínicas-. 


\section{Evaluación de la adquisición de contenidos}

Se diseñó un cuestionario de respuestas abiertas, a partir de las preguntas que guiaron el desarrollo de los contenidos y el modelo metodológico, con el objetivo de evaluar el aprendizaje de los contenidos propuestos al finalizar el CIMA (ver anexo). Posteriormente, los participantes cumplimentaron el cuestionario de conocimientos de los estudiantes, en la primera sesión del CIMA, y el mismo cuestionario en la última. (Gallardo, Pérez, Verde, y García, 2017). Las respuestas al mismo fueron categorizadas y analizadas, en aras de conocer la evolución de su nivel de conocimientos. En este sentido, la progresión del aprendizaje fue positiva, dado que las respuestas otorgadas en el cuestionario final, en más del 50\% de los sujetos, mostraron una evolución notable de los esquemas mentales, en relación a los contenidos disciplinares que debían ser adquiridos por el estudiante-. Estos resultados se representan en las figuras 3, 4, y 5:

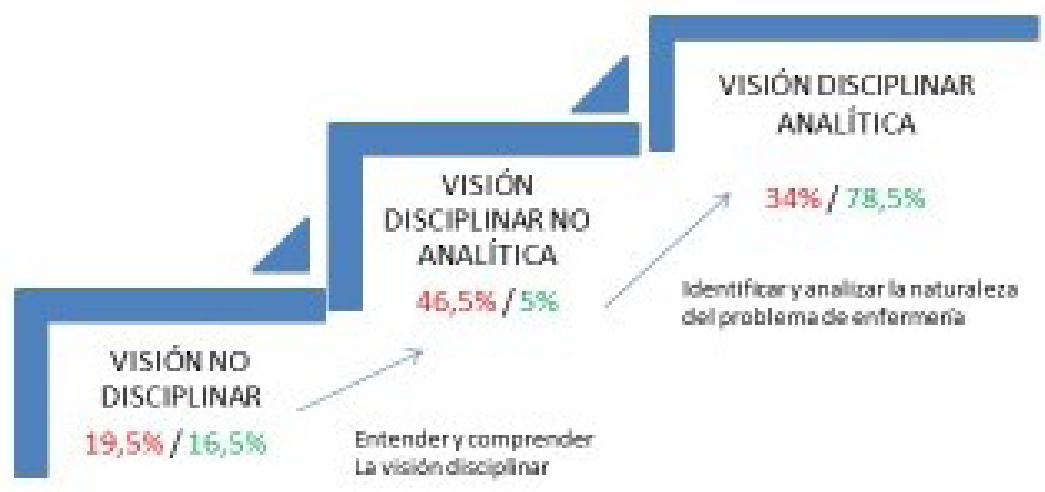

Figura 3 Progresión de respuestas a la pregunta clave

En la figura 3 se representan los modelos de pensamiento de los estudiantes y el porcentaje de alumnos que se encuentran en ese nivel en el cuestionario inicial -en color rojo-, y en el cuestionario final -en color verde-, en relación a la pregunta clave: ¿Qué tipología de problemas de enfermería existen?. En el primer nivel de la escalera, 
visión no disciplinar, los estudiantes ofrecen respuestas no vinculadas a contenidos disciplinares, sino al conocimiento general de la población. En el segundo nivel, se encuentran los estudiantes que comprenden la perspectiva bio-psico-social de la persona, y son capaces de identificar problemas de enfermería en esas áreas, aunque de forma poco analítica, y no relacionada con los modelos teóricos que guían la práctica enfermera. En el tercer nivel, los discentes son capaces de identificar y analizar la naturaleza del problema y nombrarlos aplicando modelos teóricos de enfermería.

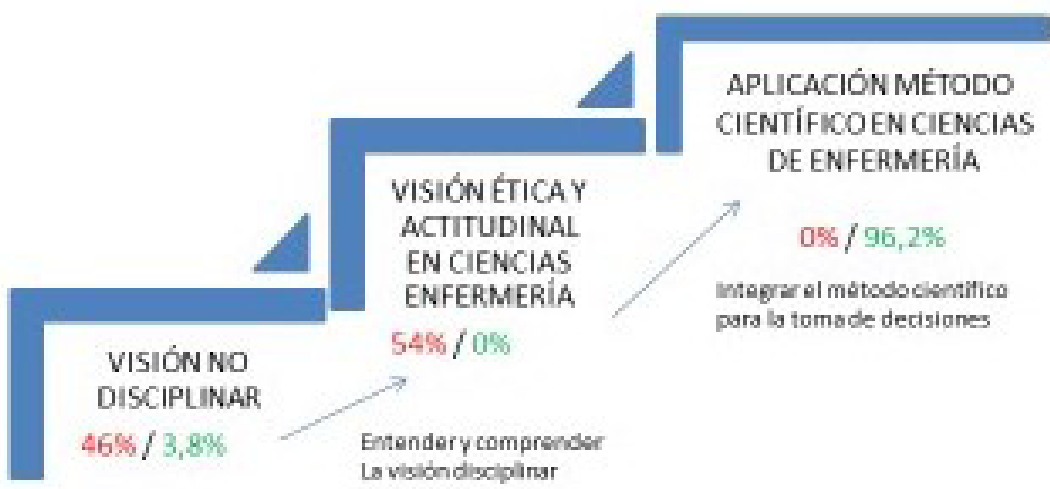

Figura 4 Progresión de respuestas a la pregunta $1 \mathrm{~A}$

De igual forma que en la figura anterior, en la figura 4 se representan los modelos de pensamiento de los estudiantes respecto a la pregunta $1 \mathrm{~A}$ : ¿Qué técnicas de valoración existen para identificar los problemas de enfermería?. En el primer nivel de la escalera, los estudiantes ofrecen respuestas no vinculadas a contenidos disciplinares. En el segundo nivel, su concepción parte de conocimientos en ciencias de enfermería, pero basados únicamente en cuestiones éticas y actitudinales, como son: la intimidad, respeto, secreto profesional, etc., -sin reflejar aspectos metodológicos disciplinares-. En el último nivel, los discentes han integrado el método científico para la toma de decisiones en ciencias de enfermería, y son capaces de comprender que deben recoger datos mediante el uso de técnicas rigurosas y específicas para ello. 


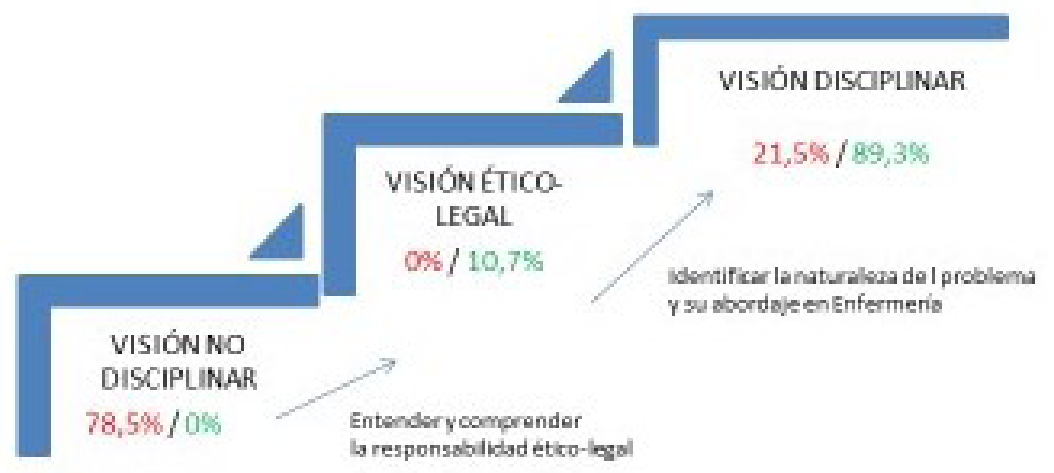

Figura 5 Progresión de respuestas a la pregunta 1 B

En la figura 5 se representan los modelos de pensamiento de los estudiantes respecto a la pregunta 1 B: ¿Cuál es el rol y la responsabilidad del profesional de enfermería en ellos?. En el primer nivel, los estudiantes ofrecen respuestas no vinculadas a contenidos disciplinares. En el segundo nivel, los alumnos creen que su responsabilidad y/o rol sobre los problemas de enfermería es únicamente de índole ético-legal. En el tercer nivel, los discentes han comprendido que, más allá de la responsabilidad ético-legal de las intervenciones enfermeras, hay un abordaje que es, en ocasiones, compartido con otros profesionales, y en otras, únicamente desde la disciplina enfermería.

A continuación, se presenta la tabla 1, que describe la progresión de los esquemas de pensamiento de cada estudiante respecto a la pregunta clave:

Jornadas de Formación e Innovación Docente del Profesorado | № 2 (2019) Esta obra se distribuye con la licencia Creative Commons Reconocimiento-NoComercial-SinObraDerivada 
Tabla 1 Evolución del esquema de pensamiento de los estudiantes respecto a la pregunta clave

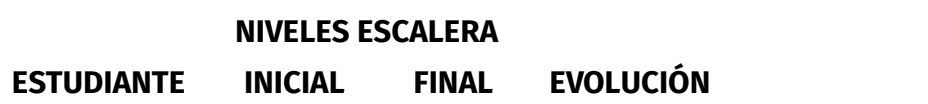

1

2

3

4

5

6

7

8

9

10

1

$2 \pi$

2

$3 \pi$

$\begin{array}{lll}-3 & 3 & \\ -1 & -3\end{array}$

$\begin{array}{llll}1 & 3\end{array}$

$\begin{array}{llll}2 & 3 & \pi\end{array}$

$\begin{array}{llll}2 & 3 & \pi\end{array}$

$\begin{array}{llll}1 & 2 & \pi\end{array}$

11

12

13

14

15

16

17

18

19

20

21

22

23

24

25

26

3

3

2

3

2

3

3

3

3

3

3

3

2

3

2

3

3

3

3

3

$\lambda$

$\pi$

$\lambda$

$\lambda$

$\pi$ 


\section{Conclusiones}

Cabe destacar que la asistencia a las sesiones fue elevada, y la valoración de los estudiantes respecto a las actividades docentes desarrolladas fue muy positiva. Así mismo, verbalizaron la necesidad de aumentar las horas de simulación de casos, con el objetivo de comprender mejor la situación de salud de la persona protagonista de los casos clínicos propuestos. Es, por tanto, un elemento a considerar en el diseño del plan docente de la asignatura. Respecto a la evaluación de la adquisición de los contenidos, los resultados de la evolución de la misma fueron positivos. Con ello, es posible concluir que el CM implementado consiguió los objetivos propuestos. Así mismo, el CM y los resultados obtenidos fueron elevados a la Coordinación de la asignatura y a la Dirección del Departamento de Enfermería, para su posterior consideración en el diseño de las actividades docentes para el próximo curso académico.

Finalmente, en este contexto de innovación docente, los principios didácticos que se extraen de la experiencia de innovación, y que deben ser aplicados en las sesiones teórico-prácticas de la asignatura son los siguientes:

El docente debe iniciar su proceso de enseñanza-aprendizaje considerando el nivel de conocimiento previo del estudiante.

Las actividades docentes deben diseñarse a partir del conocimiento base del discente, fijando como resultado de aprendizaje la adquisición de los contenidos de la asignatura.

Considerar los contenidos procedimentales y actitudinales en el diseño de actividades docentes.

Realizar una evaluación continuada del alumno, en aras de facilitar su proceso de aprendizaje y observar su evolución. 


\section{Referencias bibliográficas}

Agencia Nacional de Evaluación de la Calidad y Acreditación. (2004). Libro blanco. Título de Grado en Enfermería. ANECA.

Agencia Nacional de Evaluación de la Calidad y Acreditación. (2008). Programa de evaluación VERIFICA. Retrieved 16 November 2017, from http://www.aneca.es/ Programas-de-evaluacion/VERIFICA

Alfaro, R. (2003). Aplicación del proceso enfermero. Fomentar el cuidado en colaboración (5a). Barcelona: Masson.

Bain, K. (2007). ¿Cómo dirigen la clase? In O. Barberá (Ed.), Lo que hacen los mejores profesores universitarios (pp. 113-132). New York: Publicacions Universitat de València.

Carpenito, L. (2009). Nursing care plans \& documentation: nursing diagnoses and collaborative problems (5th ed.). Philadelphia: Lippincott Williams \& Wilkins. Retrieved from https://trove.nla.gov.au/version/38432787

Cibanal, J., y Arce, M. (2009). La relación enfermera-paciente (3a ed.). Medellín-Colombia: Editorial Universidad de Antioquia - Colección Salud Enfermería.

Departamento de Enfemería de la Universidad de Sevilla. (2019). Grado en Enfermería de la Universidad de Sevilla. Retrieved 16 October 2019, from http://www.fefp. us.es/estudios/grados/enfermeria

Departamento de Enfermería de la Universidad de Sevilla. (2019). Programa asignatura Historia, teoría y Métodos de Enfermería II. Retrieved 16 October 2019, from https://sevius.us.es/asignus/programapublicado. php?codasig $=1570016$

Fernandez, C., Navarro, M., y Luis, M. (2005). De la teoría a la práctica: el pensamiento de Virginia Henderson en el siglo XXI (3a). Mason.

Gallardo, B., Pérez, C., Verde, I., y García, E. (2017). Estilos de aprendizaje en estudiantes universitarios y enseñanza centrada en el aprendizaje. Revista ELectrónica de Investigación y Evaluación Educativa, 23(2). https://doi. org/org/10.7203/relieve.23.2.9078

Wilkinson, J. (2012). Nursing process and Critical Thinking (5th ed.). Pearson. 


\section{Anexo: Cuestionario de evaluación de conocimientos}

A continuación, se detalla un contexto clínico en el que debes llevar a cabo tu trabajo como enfermero/a. Teniendo en cuenta los datos que en él se describe, ¿podrías aportar tu opinión respecto a las dos preguntas que se formulan?

"Has sido contratado para trabajar en la Unidad de Urología del Hospital Virgen X como enfermero/a. Son las 10:00 am y ha llegado un nuevo usuario, que ingresa en una de las camas que están bajo tu responsabilidad, tras una intervención quirúrgica de vejiga. Una vez ubicado en su habitación por los celadores/as, entras en la misma para iniciar tu trabajo como enfermero/a"

a) ¿Qué herramientas podrías implementar para llevar a cabo la valoración e identificación de problemas en enfermería?

b) Una vez que has valorado a tu paciente, ¿Qué tipología de problemas de enfermería podrías identificar?

c) Tras su identificación, ¿Cuál sería tu responsabilidad, en relación a las intervenciones que lleves a cabo, en cada uno de ellos?

MUCHAS GRACIAS POR TU COLABORACIÓN

Jornadas de Formación e Innovación Docente del Profesorado | № 2 (2019) Esta obra se distribuye con la licencia Creative Commons Reconocimiento-NoComercial-SinObraDerivada 